\title{
Mechanical Properties of ZnO Nanowires Under Different Loading Modes
}

\author{
Feng $X u^{1}$, Qingqun Qin ${ }^{1}$, Ashish Mishra ${ }^{2}, Y_{i}$ Gu$^{2}$, and Yong Zhu ${ }^{1}(\bowtie)$ \\ ${ }^{1}$ Department of Mechanical and Aerospace Engineering, North Carolina State University, Raleigh, North Carolina 27695, USA \\ ${ }^{2}$ Department of Physics and Astronomy, Washington State University, Pullman, Washington 99164, USA \\ Received: 30 November 2009 / Revised: 17 January 2010 / Accepted: 8 February 2010 \\ C The Author(s) 2010. This article is published with open access at Springerlink.com
}

\begin{abstract}
A systematic experimental and theoretical investigation of the elastic and failure properties of $\mathrm{ZnO}$ nanowires (NWs) under different loading modes has been carried out. In situ scanning electron microscopy (SEM) tension and buckling tests on single $\mathrm{ZnO}$ NWs along the polar direction [0001] were conducted. Both tensile modulus (from tension) and bending modulus (from buckling) were found to increase as the NW diameter decreased from 80 to $20 \mathrm{~nm}$. The bending modulus increased more rapidly than the tensile modulus, which demonstrates that the elasticity size effects in $\mathrm{ZnO}$ NWs are mainly due to surface stiffening. Two models based on continuum mechanics were able to fit the experimental data very well. The tension experiments showed that fracture strain and strength of $\mathrm{ZnO}$ NWs increased as the NW diameter decreased. The excellent resilience of $\mathrm{ZnO}$ NWs is advantageous for their applications in nanoscale actuation, sensing, and energy conversion.
\end{abstract}

\section{KEYWORDS}

$\mathrm{ZnO}$ nanowire, mechanical property, size effect, Young's modulus, fracture

\section{Introduction}

As an important semiconducting and piezoelectric material with a large exciton binding energy and a wide band gap [1], ZnO nanowires (NWs) have found broad applications ranging from nanoelectromechanical systems (NEMS) [2] to nanosensors [3, 4] to nanogenerators [5, 6]. Mechanical properties of $\mathrm{ZnO} N W s$, including both elasticity and fracture, are of critical relevance to the design and reliability of these devices. In many devices such as nanogenerators $[5,6], \mathrm{ZnO}$ NWs undergo different mechanical loadings such as tension, bending or buckling, and it is therefore important to study the effect of loading mode on their mechanical properties.

Mechanical characterization of individual NWs is important but challenging. A number of methods have been developed for mechanical testing of $\mathrm{ZnO}$ NWs including resonance in scanning or transmission electron microscopes (SEM/TEM) [7,8], bending or contact resonance using atomic force microscopy (AFM) [9-11], uniaxial tension in SEM or TEM [12, 13], and nanoindentation [14]. In particular, in situ SEM/TEM tensile testing of NWs enabled by microelectromechanical systems (MEMS) has attracted a lot of recent attention [12, 15, 16]. The experimental results, however, showed both scatter and inconsistency [17]. Some researchers observed that the Young's

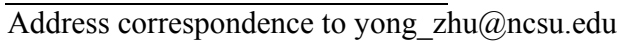


modulus increased with decreasing NW diameter $[7,9,12]$, while others reported essentially no size dependence $[8,10]$. Computational studies including continuum mechanics and molecular dynamics (MD) have exhibited similar disagreement. Various mechanisms such as bulk nonlinear elasticity [18, 19], surface bond saturation [20], surface reconstruction $[3,7,12]$, and others [21] have been proposed to explain the elasticity size effects in $\mathrm{ZnO}$ NWs. Yet few experiments have conclusively supported one or other of these mechanisms.

In addition to the experimental errors, one possible reason for the observed discrepancy between experimental measurements of the Young's modulus of $\mathrm{ZnO}$ NWs is because different loading modes (bending, resonance, tension) have been used. Surface effects are expected to be more pronounced under flexural loading (i.e., bending or resonance) than tension as surfaces carry the most stress in the former $[22,23]$. Therefore, it is important to conduct quantitative experiments under different loading modes while keeping the remaining experimental conditions constant. The objectives are twofold: (1) to explain the large discrepancy in experimental results in the literature, and (2) to elucidate the underlying mechanism(s) of the size effects, i.e., the relative importance of the surface and the core of NWs, especially when integrated with computational studies. So far only one study compared the Young's moduli of $\mathrm{ZnO} \mathrm{NWs}$ under tension and resonance and found considerable difference in the size effects, albeit with very different setups/conditions in the two experimental methods [24].

The majority of the reported studies focused only on the elastic properties (i.e., Young's modulus) of ZnO NWs; fewer studies have investigated their fracture under tension or bending [10, 11, 25, 26]. No work has been reported on the postbuckling behavior of $\mathrm{ZnO}$ NWs despite its relevance to NEMS, nanogenerators, and other applications. Furthermore, failure of $\mathrm{ZnO} \mathrm{NWs}$ under different loading modes has not been compared.

In this paper, we report size effects on the elasticity and fracture of $\mathrm{ZnO}$ NWs under tension and bending. Bending was imposed in the buckling tests. Both types of tests were conducted in situ in SEM using the same setup (a nanomanipulator probe as actuator and an AFM cantilever as load sensor). Both tensile modulus (from tension) and bending modulus (from buckling) were found to increase as the NW diameter decreased from 80 to $20 \mathrm{~nm}$. The bending modulus increased more rapidly than the tensile modulus. The fracture strain and fracture strength under tension increased as the NW diameter decreased. ZnO NWs were found to sustain very large bending deformation during postbuckling.

\section{Experimental}

The NWs used in this study were synthesized by the vapor-liquid-solid method on $\mathrm{Si} / \mathrm{SiO}_{2}$ substrates with $\mathrm{Au}$ colloids as the catalysts [27]. A mixture of $\mathrm{ZnO}$ (Alfa Aesar, 99.999\%) and graphite (Alfa Aesar, 99.9995\%) powders in a quartz boat was used as the $\mathrm{Zn}$ source. This quartz boat was heated to $950^{\circ} \mathrm{C}$ by a local heater, and the generated $\mathrm{Zn}$ vapor was carried by a flow of Ar to the growth zone, where $\mathrm{O}_{2}$ was injected to enable the NW growth. The temperature of the growth zone was held at $820^{\circ} \mathrm{C}$ during the growth under a constant pressure of 7.5 Torr. After the growth (the duration of which was $30 \mathrm{~min}$ ), the reactor was first pumped to the base pressure and then was cooled down under a flow of Ar.

All the tension and buckling tests were performed inside an SEM (JEOL 6400F). A nanomanipulator (Klocke Nanotechnik, Germany) with $1 \mathrm{~nm}$ resolution and $1 \mathrm{~cm}$ travel range in three orthogonal directions was used to pick up protruding NWs from the Si wafer following the procedure outlined in Zhu and Espinosa [16] and to carry out the tension and buckling experiments. For tension tests, an AFM chip (ORC8-10, Veeco) with two silicon nitride cantilevers on one side was mounted on a sample holder. A detailed description of the experimental process for tension tests can be found elsewhere [28]. The buckling tests were carried out similarly, but the cantilever used in the buckling tests was much softer (OBL-10, Veeco) than that used in the tension tests. After the NW was clamped between the nanomanipulator tip and the AFM cantilever, the NW was continously pushed until beyond the critical force for buckling. The postbuckling shape of the NW can be clearly seen. Similarly, the 
force and compression data were directly obtained from the images.

The cantilever stiffness was calibrated using the Sader method [29]. The measured values were $0.70 \mathrm{~N} / \mathrm{m} \pm$ $0.05 \mathrm{~N} / \mathrm{m}$ and $0.018 \pm 0.002 \mathrm{~N} / \mathrm{m}$ for the cantilevers in tension and buckling tests, respectively. The loads on the NW were calculated from the cantilever deflections, which were obtained in the SEM images with reference to a stationary feature. The stationary feature is a neighboring cantilever on the same chip and is out of focus in the SEM images; see the Electronic Supplementary Material (ESM) for more details. For tension tests, the cantilever deflection was measured in images with lower magnification, where a resolution of half a pixel translated to $9.6 \mathrm{~nm}$. Therefore, the force resolution was $6.72 \mathrm{nN} \pm 0.48 \mathrm{nN}$. For NWs with diameters ranging from 20 to $80 \mathrm{~nm}$, the stress resolution ranged from 21.4 to $1.3 \mathrm{MPa}$. The NW elongation was measured in images with higher magnification (the image possesses $2000 \times 1600$ pixels and an NW typically spans $~ 1500-1800$ pixels in length). Therefore, the strain resolution was about $0.03 \%$. For buckling tests, the cantilever deflection and the axial displacement of the NW were measured from the same images, with a resolution of half a pixel translating to $3.9 \mathrm{~nm}$. The force resolution was $0.07 \mathrm{nN} \pm 0.01 \mathrm{nN}$.

\section{Results and discussion}

\subsection{Experimental results}

Figure 1(a) shows an SEM image of the $\mathrm{ZnO} \mathrm{NW}$ sample on the silicon substrate. Figure 1(b) displays the TEM image of a $\mathrm{ZnO} \mathrm{NW}$ with diameter around
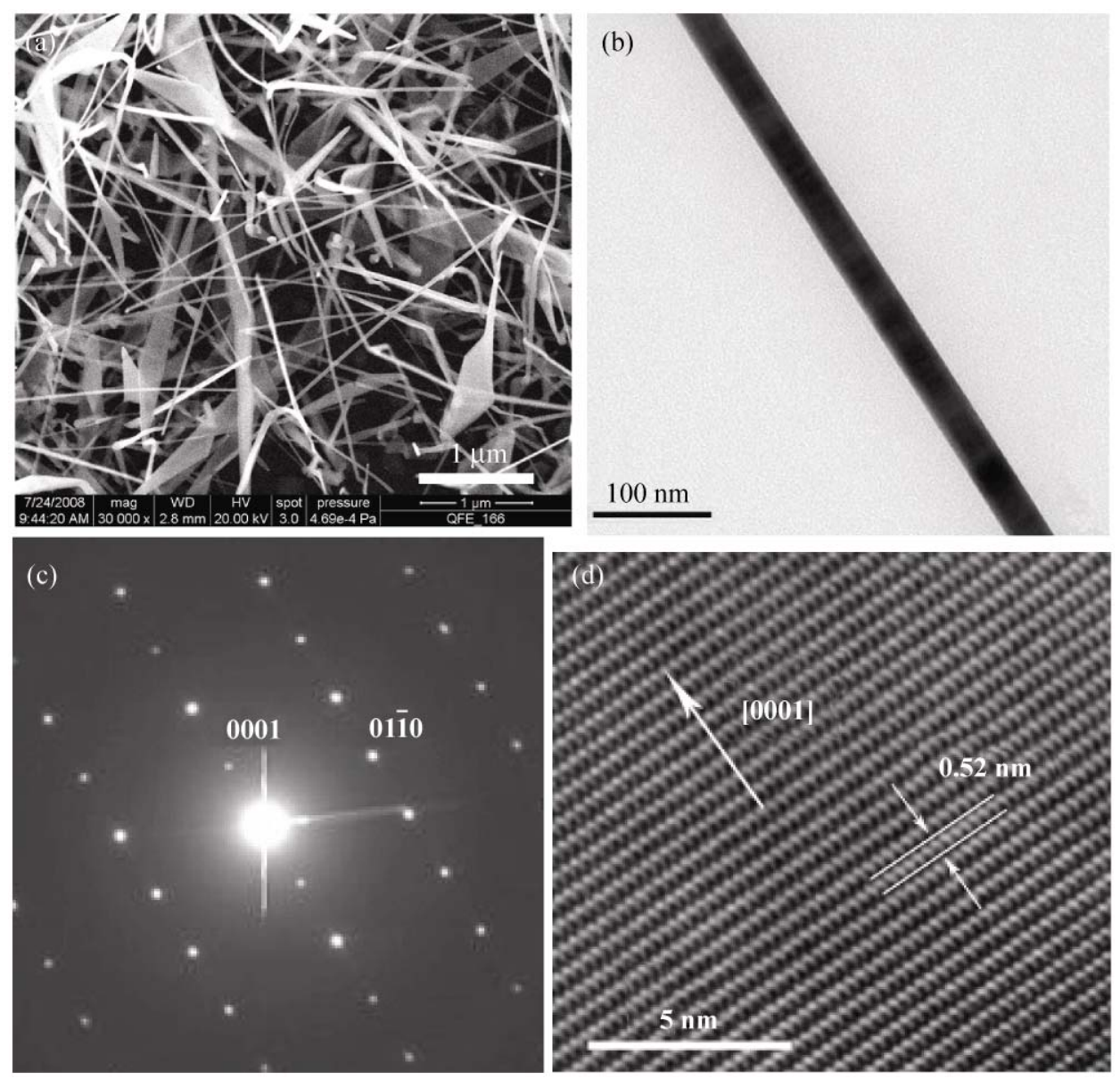

Figure 1 (a) Typical SEM image of the ZnO NWs with diameter of $50 \mathrm{~nm}$. (b) TEM image of a single ZnO NW. (c) The corresponding SAED pattern of the ZnO NW. (d) High-resolution TEM image of the NW 
$30 \mathrm{~nm}$. It can be seen that the NW is not only straight but also uniform in diameter along the growth direction. The corresponding selected area electron diffraction (SAED) pattern in Fig. 1(c) and highresolution TEM image in Fig. 1(d) indicate that the wires are single-crystalline, with a growth direction along the [0001] axis.

During the tensile test, a series of SEM images were taken to measure both force and elongation of the NWs. A few typical images from a NW with diameter of $20 \mathrm{~nm}$ are shown in Figs. 2(a)-2(c). It can be seen that two ends of the NW are clamped on the nanomanipulator tip (left) and the AFM cantilever (right), respectively. Figure 2(a) is the image prior to loading. Figures 2(b) and 2(c) show the NW under the tensile loads of 2.13 and $3.05 \mu \mathrm{N}$, respectively.
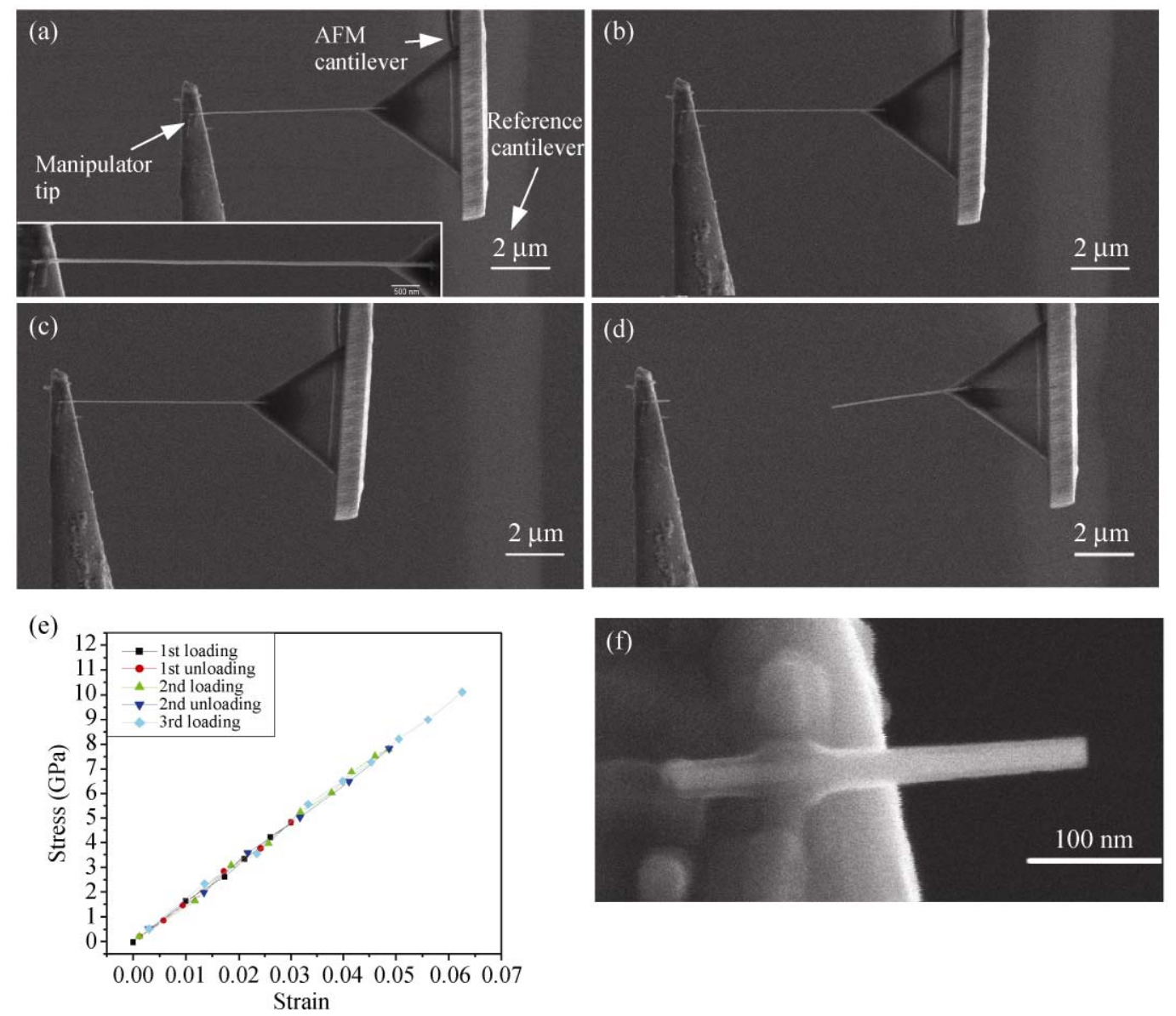

Figure $2(\mathrm{a}-\mathrm{c})$ A series of SEM images taken during the tensile test for an NW with diameter of $20 \mathrm{~nm}$. Inset of (a): high-resolution SEM image of the NW used for strain measurements. (d) SEM image showing that fracture occurs on the NW when the load was applied to a certain value. (e) A typical stress-strain response of the specimens with diameter of $20 \mathrm{~nm}$ under repeated loading and unloading. The errors for the stress and strain are $\pm 0.72 \mathrm{GPa}$ and $\pm 0.004 \mathrm{GPa}$, respectively. (f) Enlarged SEM image of the broken end of the NW on the probe tip
The NW was tested in a few loading and unloading cycles until fracture, as shown in Fig. 2(d). Figure 2(e) shows the stress-strain response of this NW. The Young's modulus was measured to be approximately $169 \mathrm{GPa}$, which is higher than the bulk value of $\mathrm{ZnO}$ in the [0001] direction ( 140 GPa) [12]. It can also be seen that the loading and unloading processes followed almost the same path showing a linear elastic behavior. No residual plastic deformation was observed when the NW was totally unloaded. This observation is corroborated by the enlarged image of the broken end shown in Fig. 2(f). The broken end appears flat and no obvious diameter reduction or necking can be seen. No NW slippage was observed at either end, indicating that the carbon deposition clamp was strong enough for testing $\mathrm{ZnO} \mathrm{NWs}$ with diameters up to 
$80 \mathrm{~nm}$ [28]. Tension tests of NWs with different diameters were all conducted following the same procedure with multiple loading/unloading cycles and all the NWs showed linear elastic behavior.

In situ SEM buckling tests of $\mathrm{ZnO} \mathrm{NWs}$ were conducted in a similar fashion [30]. In order to make a comparison with the tensile tests, NWs with similar diameters were intentionally sought and used for the buckling tests. Figure 3(a) is an SEM image of a $\mathrm{ZnO}$ NW with diameter of $46 \mathrm{~nm}$. Both ends of the NW were clamped by the method of electron beam induced deposition (EBID). The buckling process accompanied by the shape change of the NW under an incrementally increasing compressive load is displayed in Figs. 3(b)-3(e). Figure 3(b) shows the straight NW prior to the compressive loading. When the force reached the critical value, the NW buckled, as can be seen from Fig. 3(c). Figures 3(d) and 3(e) show progressive, symmetric postbuckling deformation of the NW. The variation of applied force with the axial displacement of the NW is plotted in Fig. 3(f). Initially, the force increased rapidly with the axial displacement. When the force reached the critical force $\left(P_{\mathrm{cr}}\right)$ for buckling, the load-displacement curve became nearly flat. $P_{\mathrm{cr}}$ was determined to be $62 \mathrm{nN}$ in this case.

According to Euler's formula, the buckling force $P_{\text {cr }}$ of an ideal elastic column is given by

$$
P_{\text {cr }}=\frac{\pi^{2} E I}{L_{\mathrm{e}}^{2}}
$$

where $E$ is the Young's modulus and $I$ is the moment of inertia of a $\mathrm{ZnO} \mathrm{NW}$. Assuming the cross section of the NW is a circular shape (as will be explained later), $I$ can be expressed as $I=\pi R^{4} / 4$, where $R$ is the
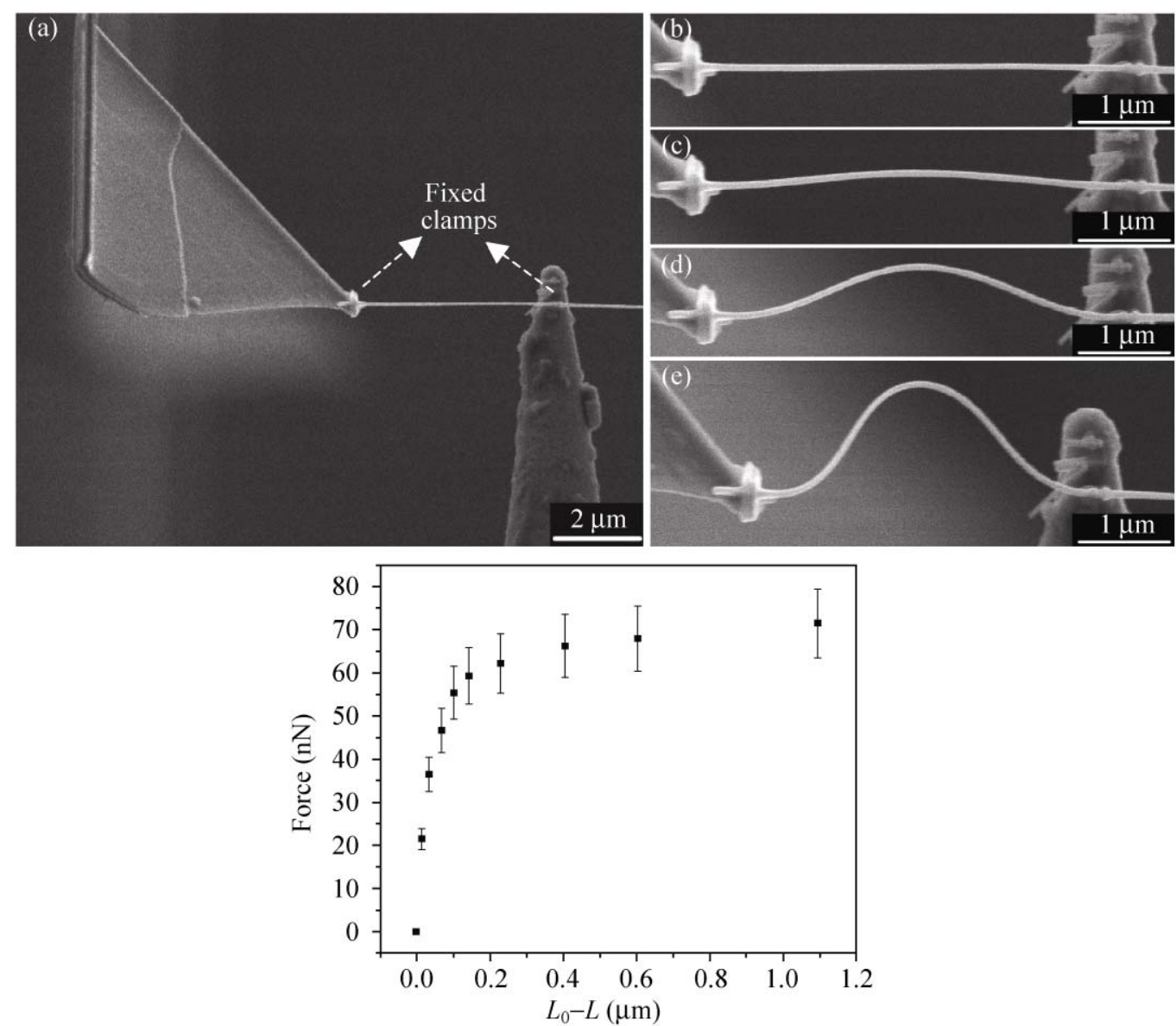

(f)

Figure 3 (a) SEM image showing the buckling test for an NW with diameter of $46 \mathrm{~nm}$. (b-e) A series of enlarged SEM images of the NW under the application of a continuously increasing compressive load. (f) The corresponding plot of the applied force versus the axial displacement of the NW 
radius of the NW. Note that in buckling tests, the Young's modulus is a bending modulus, as it is a measure of the structural resistance to bending deformation following the buckling instability. The effective length $L_{\mathrm{e}}=0.5 \mathrm{~L}$ for a fixed-fixed boundary condition (as in our experiments), where $L$ is the actual length of the column. According to this analysis, the Young's modulus of the NW shown in Fig. 3 was calculated to be $151 \mathrm{GPa}$.

\subsection{Discussion of size effects}

To study the size effects on the mechanical properties of $\mathrm{ZnO}$ NWs, seven NWs were tested under tension tests and seven NWs under buckling tests. The NWs ranged from 20 to $80 \mathrm{~nm}$ in diameter. Figure 4(a) shows the calculated Young's modulus values for all of the tested NWs as a function of size. The errors arise mainly from the measurement of NW diameters. It is evident that the Young's modulus of $\mathrm{ZnO}$ NWs from both tension and buckling tests show the stiffening trend, i.e., it increases with decreasing diameter.

However, our data also show a loading-modedependent size effect. The disparity in the Young's moduli under different loading modes (tension vs. buckling here) becomes larger as the NW diameter decreases. When the NW diameters are around $80 \mathrm{~nm}$, the Young's moduli from both tension and buckling are close to the bulk modulus. However, when the diameters decrease to $20 \mathrm{~nm}$, the modulus values obtained from the tension tests are around $170 \mathrm{GPa}$, while those from the buckling tests are more than 200 GPa. It is clear that the Young's modulus obtained from the buckling tests exhibits stronger size effects in comparison to the tension tests.

Several mechanisms have been proposed to explain the size effects in the elastic behavior of $\mathrm{ZnO}$ nanostructures, i.e., the increase in the Young's modulus with the decrease in NW diameter. Kulkarni et al. [18] and Cao et al. [19] predicted that the nonlinear elastic response of the NW core (interior) plays the major role in determining the elastic modulus of NWs. In contrast, Zhang and Huang [20] found that the surface bond saturation resulting from an increased electron density, rather than bulk nonlinear elastic effects, may be responsible for the size effects. Recently, Agrawal et al. [12], through a combined experimental/simulation study, showed that elastic stiffening in $\mathrm{ZnO}$ NWs was caused by a decrease in surface interatomic spacing due to surface reconstruction, in agreement with Chen et al. [7].

The observed size effects in Fig. 4(a) can be useful in assessing the relative importance of the surface and the core elasticity. For NWs under bending (including buckling and resonance), the surfaces carry the largest stress and strain; by contrast, NWs subjected to uniaxial tension undergo uniform stress across the cross section. The Young's modulus measured in both loading conditions is in fact the average between the surface and the core. If the bulk nonlinear elasticity dominates the stiffening size effects (i.e., the core modulus is larger than the bulk value), the average modulus of $\mathrm{ZnO}$ NWs measured in tension tests is larger than that obtained from bending tests; if the surface contributes the most to the size effects (i.e., the surface modulus is larger than the bulk value), the average modulus measured in bending tests is larger. Our experimental results clearly confirmed the latter; that is, the surface elasticity plays the major role in the stiffening size effects in $\mathrm{ZnO}$ NWs.

To further quantify the surface and core elasticity, our results were fitted to two existing models: the core-surface model (or Miller-Shenoy model) [22] and core-shell model [7], as shown in the inset of Fig. 4(a). Again we assume the NW cross section is circular. The core-surface model is a continuum mechanics approach that embodies the surface effect [31-33]. The model essentially assumes that an NW consists of a core with elastic modulus $E_{\mathrm{c}}$ and a surface (zero thickness) with so-called surface elastic modulus $S$ (the unit is $\mathrm{Pa} \cdot \mathrm{m}$ ). Under tension, the measured (or effective) Young's modulus $E$ is given by

$$
E=E_{\mathrm{c}}+4 \frac{S}{D}
$$

where $D$ is the diameter of the circular cross section. Under bending,

$$
E=E_{\mathrm{c}}+8 \frac{S}{D}
$$

In the core-shell model, the NW consists of a core with 
elastic modulus $E_{\mathrm{c}}$ and a shell with elastic modulus $E_{\mathrm{s}}$. Under tension,

$$
E=E_{\mathrm{c}}\left[1+4\left(\frac{E_{\mathrm{s}}}{E_{\mathrm{c}}}-1\right)\left(\frac{r_{\mathrm{s}}}{D}-\frac{r_{\mathrm{s}}^{2}}{D^{2}}\right)\right]
$$

where $D$ is the outer diameter of the circular cross section and $r_{\mathrm{s}}$ is the shell thickness.

Under bending,

$$
E=E_{\mathrm{c}}\left[1+8\left(\frac{E_{\mathrm{s}}}{E_{\mathrm{c}}}-1\right)\left(\frac{r_{\mathrm{s}}}{D}-3 \frac{r_{\mathrm{s}}^{2}}{D^{2}}+4 \frac{r_{\mathrm{s}}^{3}}{D^{3}}-2 \frac{r_{\mathrm{s}}^{4}}{D^{4}}\right)\right]
$$

Derivations of the above formulae are given in the ESM. The tensile results are prone to fewer errors than the buckling results; therefore, both models were fitted to the tensile data following Eqs. (2) and (4), and then the fitting parameters were substituted into Eqs. (3) and (5) to compare with the bending data. In the core-surface model, it was found that the core elastic modulus $E_{\mathrm{c}}=115 \mathrm{GPa}$ and the surface elastic modulus $S=267 \mathrm{~Pa} \cdot \mathrm{m}$; in the core-shell model, $E_{\mathrm{c}}=$ 114.7 GPa, the shell elastic modulus $E_{\mathrm{s}}=244.4 \mathrm{GPa}$, and the shell thickness $r_{\mathrm{s}}=2.42 \mathrm{~nm}$. The fitting curves are plotted in Fig. 4(a) in addition to the experimental data. Both models offered excellent fitting to the tensile data, but slightly overestimated the buckling data. Additionally both models agreed very well with each other. It is noted, however, that the obtained core elastic modulus is lower than the bulk value (140 GPa), which appears contradictory. In fact, the Young's modulus of the core is not necessarily equal to the bulk value as a result of the surface stress [33]. Recent MD simulations showed that a core and a shell with different Young's moduli exist in $\mathrm{ZnO}$ NWs; the Young's moduli of the core and of the shell are lower and higher than the bulk value, respectively [12]. The elastic modulus varies continuously from the core to the surface as a result of energy minimization $[12,24]$. Both models used in our work are first-order approximations (assuming step-like modulus distribution in the core and the shell) that at the least explain our experimental results reasonably well. Numerical simulations are required to further explain the effect of loading modes.

One important remark concerns the cross sectional shape of the ZnO NWs used in the data analysis as well as in the fitting process. It is known that [0001]-oriented $\mathrm{ZnO}$ NWs possess hexagonal cross sections. In the case of an edge facing the electron beam, treating the cross section as a circle overestimates the cross sectional area and moment of inertia; in the case of a vertex facing the electron beam, such a treatment underestimates these quantities. In the experiments, the NW cross sections could be oriented randomly relative to the electron beam. Therefore, statistically speaking, it is reasonable to use circular cross sections for ZnO NWs and other types of NWs with similar cross sections. This treatment does not introduce systematic errors, but it does introduce random errors that contribute to the total experimental errors.

Figures 4(b) and 4(c) show the variation of fracture strain and fracture strength with the diameter of the NWs from the tension tests. Both fracture strain and fracture strength were found to increase with decreasing NW diameter. These values were much higher than the corresponding values for bulk $\mathrm{ZnO}$, which are typically smaller than $1 \%$ [13] and $200 \mathrm{MPa}$ [34], respectively. Similar size effects on the fracture strain have been reported by Desai and Haque [13]. However, their fracture strain was more than $10 \%$ for NWs with diameters less than $300 \mathrm{~nm}$; in our experiments, the largest fracture strain is about $6.1 \%$ for the smallest NW with diameter of $20 \mathrm{~nm}$. Atomistic simulations predicted a phase transformation from the wurtzite structure to a body centered-tetragonal structure with four-atom rings (BCT-4) for $\mathrm{ZnO}$ nanorods [35]. But such a phase transformation was not observed in our experiments, as can be seen from the repeated linear elastic behavior during the loading-unloading process. Perhaps due to the presence of defects, the maximum fracture strain in our experiments is less than the strain value of $7.5 \%$ at which the phase transformation was predicted to occur [35]. Our experimental observation is consistent with recent in situ TEM tensile experiments by Agrawal et al. [26]. For the fracture strength, our values ranging from 4.10 to $10.32 \mathrm{GPa}$ are comparable to those obtained from bending experiments by Wen et al. [10]. These authors also found a similar diameter 


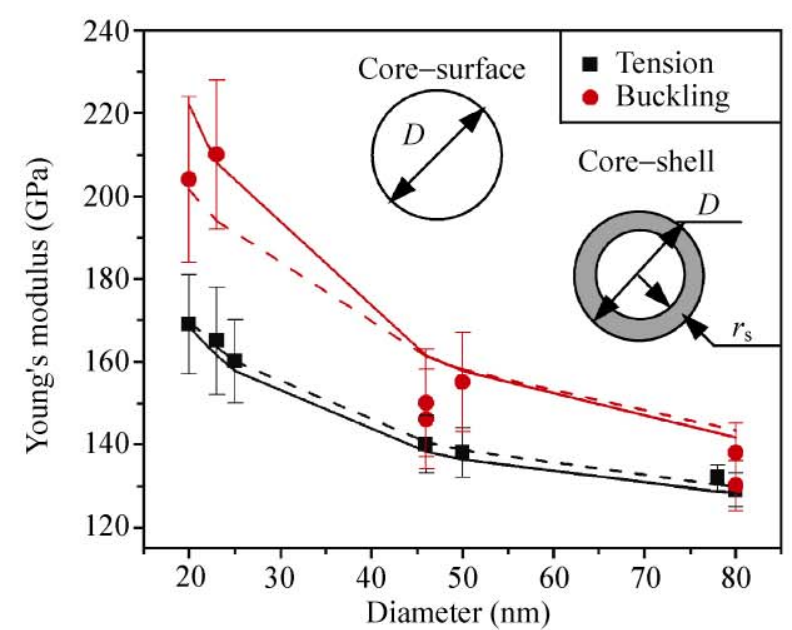

(a)

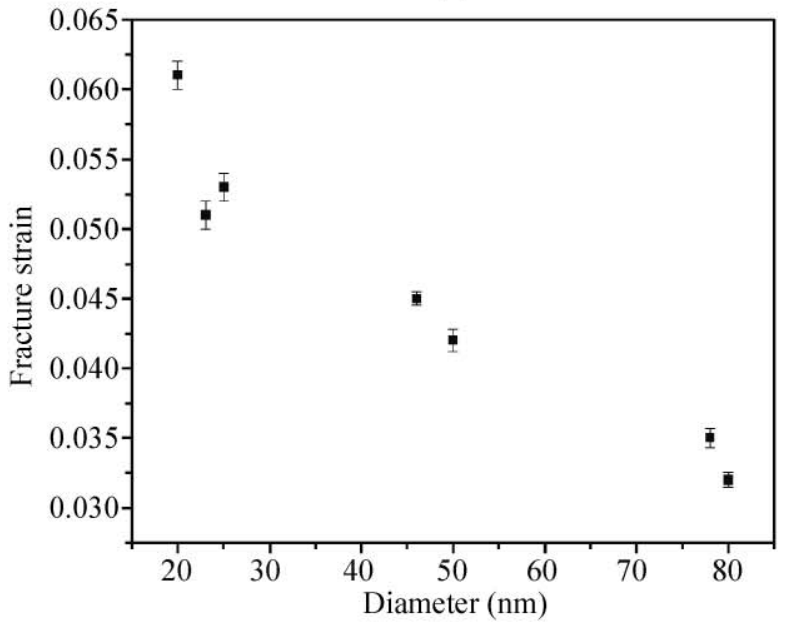

(b)

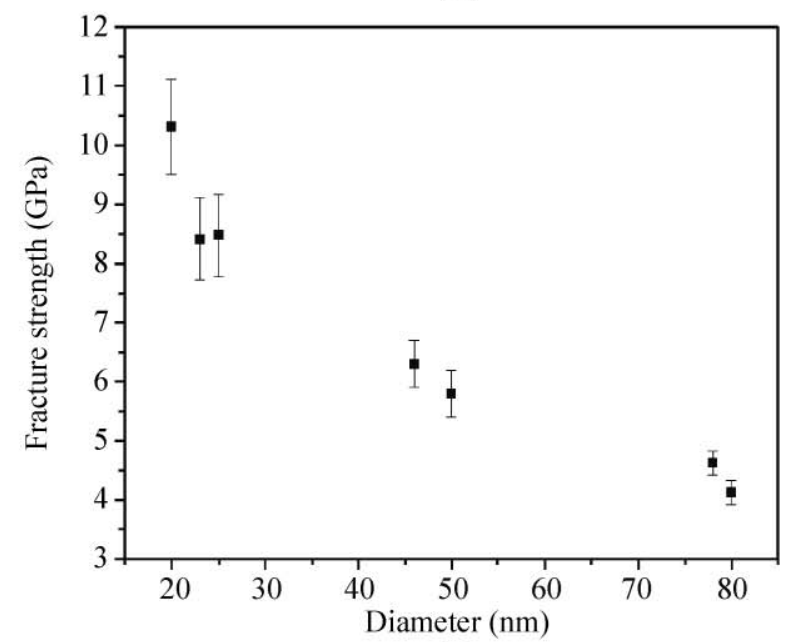

(c)

Figure 4 (a) Plots of variation of Young's modulus with diameter of $\mathrm{ZnO} \mathrm{NWs}$ from both tension and buckling tests. The fitting curves from the core-surface model (solid lines) and the core-shell model (dashed lines) are also plotted. The inset shows schematic views of both models. Variation with diameter of (b) fracture strain and (c) fracture strength of $\mathrm{ZnO} N W s$ from tension tests dependence of the fracture strength of $\mathrm{ZnO} N W s$. One possible reason for the size effects on the fracture strain and strength from tension is due to the bond-length contraction of surface atoms in $\mathrm{ZnO}$ NWs as a result of surface reconstruction [12, 13, 21]. The bond-length contraction results in strengthening of the bond. Hence, the binding energy of surface atoms tends to be higher than that of bulk atoms. With the increasing surface-to-volume ratio at the nanoscale, this mechanism could lead to higher fracture strain and strength values [13]. Another possible reason is the presence of defects in the NWs. For NWs with smaller size, the number of defects is reduced, consistent with the increase in the fracture strain and strength.

\section{Conclusions}

We have presented an experimental approach to evaluate the elastic and failure properties of $\mathrm{ZnO} N W \mathrm{~s}$ under different loading modes. In situ SEM tension and buckling tests on single $\mathrm{ZnO} \mathrm{NWs}$ along the polar direction [0001] were conducted. Both tensile modulus (from tension) and bending modulus (from buckling) were found to increase as the NW diameter decreased from 80 to $20 \mathrm{~nm}$. The bending modulus increased more rapidly than the tensile modulus, which demonstrates that the elasticity size effects in $\mathrm{ZnO}$ NWs are mainly due to surface stiffening. Both the core-surface model and the core-shell model were able to fit the experimental data very well. The tension experiments also showed that the fracture strain and strength of $\mathrm{ZnO} N W$ s increased as the NW diameter decreased. The excellent resilience of $\mathrm{ZnO}$ NWs is advantageous for their applications in NEMS, nanosensors and nanogenerators. Finally, the buckling technique presented in this work can be extended to evaluate the elastic modulus of other NWs under bending stress.

\section{Acknowledgements}

This work was supported by the National Science Foundation under Award No. CMMI-0826341 and a Faculty Research and Professional Development Award from North Carolina State University. 
Electronic Supplementary Material: The experimental procedure for the use of the AFM cantilever as a load sensor and the derivation of continuum models of elasticity size effects are available in the online version of this article at http://dx.doi.org/10.1007/s12274-0101030-4 and are accessible free of charge.

Open Access: This article is distributed under the terms of the Creative Commons Attribution Noncommercial License which permits any noncommercial use, distribution, and reproduction in any medium, provided the original author(s) and source are credited.

\section{References}

[1] Wang, Z. L. Zinc oxide nanostructures: Growth, properties and applications. J. Phys.: Condens. Mater. 2004, 16, R829-R858.

[2] Zhou, J.; Fei, P.; Gao, Y. F.; Gu, Y. D.; Liu, J.; Bao, G.; Wang, Z. L. Mechanical-electrical triggers and sensors using piezoelectric micowires/nanowires. Nano Lett. 2008, 8, 2725-2730.

[3] Yuan, Q. Z.; Zhao, Y. P.; Li, L. M.; Wang, T. H. Ab initio study of ZnO-based gas-sensing mechanisms: Surface reconstruction and charge transfer. J. Phys. Chem. C 2009, 113, 6107-6113.

[4] Wan, Q.; Li, Q. H.; Chen, Y. J.; Wang, T. H.; He, X. L.; Li, J. P.; Lin, C. L. Fabrication and ethanol sensing characteristics of $\mathrm{ZnO}$ nanowire gas sensors. Appl. Phys. Lett. 2004, 84, 3654-3656.

[5] Wang, Z. L.; Song, J. H. Piezoelectric nanogenerators based on zinc oxide nanowire arrays. Science 2006, 312, 242-246.

[6] Yang, R. S.; Qin, Y.; Dai, L. M.; Wang, Z. L. Power generation with laterally-packaged piezoelectric fine wires. Nat. Nanotechnol. 2009, 4, 34-39.

[7] Chen, C. Q.; Shi, Y.; Zhang, Y. S.; Zhu, J.; Yan, Y. J. Size dependence of Young's modulus in $\mathrm{ZnO}$ nanowires. Phys. Rev. Lett. 2006, 96, 075505.

[8] Bai, X. D.; Gao, P. X.; Wang, Z. L.; Wang, E. G. Dual-mode mechanical resonance of individual $\mathrm{ZnO}$ nanobelts. Appl. Phys. Lett. 2003, 82, 4806.

[9] Stan, G.; Ciobanu, C. V.; Parthangal, P. M.; Cook, R. F. Diameter-dependent radial and tangential elastic moduli of ZnO nanowires. Nano Lett. 2007, 7, 3691-3697.

[10] Wen, B. W.; Sader, J. E.; Boland, J. J. Mechanical properties of ZnO nanowires. Phys. Rev. Lett. 2008, 101, 175502.

[11] Hoffmann, S.; Ostlund, F.; Michler, J.; Fan, H. J.; Zacharias,
M.; Christiansen, S. H.; Ballif, C. Fracture strength and Young's modulus of $\mathrm{ZnO}$ nanowires. Nanotechnology 2007, 18,205503

[12] Agrawal, R.; Peng, B.; Gdoutos, E. E.; Espinosa, H. D. Elasticity size effects in $\mathrm{ZnO}$ nanowires-A combined experimental-computational approach. Nano Lett. 2008, 8, 3668-3674.

[13] Desai, A. V.; Haque, M. A. Mechanical properties of $\mathrm{ZnO}$ nanowires. Sensor. Actuat. A: Phys. 2007, 134, 169-176.

[14] Ni, H.; Li, X. D. Young's modulus of $\mathrm{ZnO}$ nanobelts measured using atomic force microscopy and nanoindentation techniques. Nanotechnology 2006, 17, 3591-3597.

[15] Zhu, Y.; Moldovan, N.; Espinosa, H. D. A microelectromechanical load sensor used for in situ electron and X-ray microscopy tensile testing of nanostructures. Appl. Phys. Lett. 2005, 86, 013506.

[16] Zhu, Y.; Espinosa, H. D. An electro-mechanical material testing system for in situ electron microscopy and applications. Proc. Natl. Acad. Sci. U.S.A. 2005, 102, 14503-14508.

[17] Park, H. S.; Cai, W.; Espinosa, H. D.; Huang, H. Mechanics of crystalline nanowires. MRS Bull. 2009, 34, 178-183.

[18] Kulkarni, A. J.; Zhou, M.; Ke, F. J. Orientation and size dependence of the elastic properties of zinc oxide nanobelts. Nanotechnology 2005, 16, 2749-2756.

[19] Cao, G. X.; Chen, X. Energy analysis of size-dependent elastic properties of $\mathrm{ZnO}$ nanofilms using atomistic simulations. Phys. Rev. B 2007, 76, 165407.

[20] Zhang, L. X.; Huang, H. C. Young's moduli of $\mathrm{ZnO}$ nanoplates: Ab initio determinations. Appl. Phys. Lett. 2006, $89,183111$.

[21] Liu, X. J.; Li, J. W.; Zhou, Z. F.; Yang, L. W.; Ma, Z. S.; Xie, G. F.; Pan, Y.; Sun, C. Q. Size-induced elastic stiffening of $\mathrm{ZnO}$ nanostructures: Skin-depth energy pinning. Appl. Phys. Lett. 2009, 94, 131902.

[22] Miller, R. E.; Shenoy, V. B. Size-dependent elastic properties of nanosized structural elements. Nanotechnology 2000, 11, 139-147.

[23] McDowell, M. T.; Leach, A. M.; Gall, K. Bending and tensile deformation of metallic nanowires. Model. Simul. Mater. Sci. Eng. 2008, 16, 045003.

[24] He, M. R.; Shi, Y.; Zhou, W.; Chen, J. W.; Yan, Y. J.; Zhu, $\mathrm{J}$. Diameter dependence of modulus in zinc oxide nanowires and the effect of loading mode: In situ experiments and universal core-shell approach. Appl. Phys. Lett. 2009, 95, 091912.

[25] Chen, C. Q.; Zhu, J. Bending strength and flexibility of $\mathrm{ZnO}$ nanowires. Appl. Phys. Lett. 2007, 90, 043105. 
[26] Agrawal, R.; Peng, B.; Gdoutos, E.; Espinosa, H. D. Experimental-computational investigation of $\mathrm{ZnO}$ nanowires strength and fracture. Nano Lett. 2009, 9, 4177-4183.

[27] Soudi, A.; Khan, E. H.; Dickinson, J. T.; Gu, Y. Observation of unintentionally incorporated nitrogen-related complexes in $\mathrm{ZnO}$ and GaN nanowires. Nano Lett. 2009, 9, 1844-1849.

[28] Zhu, Y.; Xu, F.; Qin, Q. Q.; Fung, W. Y.; Lu, W. Mechanical properties of vapor-liquid-solid synthesized silicon nanowires. Nano Lett. 2009, 9, 3934-3939.

[29] Sader, J. E.; Chon, J. W. M.; Mulvaney, P. Calibration of rectangular atomic force microscope cantilevers. Rev. Sci. Instrum. 1999, 70, 3967-3969.

[30] Hsin, C. L.; Mai, W. J.; Gu, Y. D.; Gao, Y. F.; Huang, C. T.; Liu, Y. Z.; Chen, L. J.; Wang, Z. L. Elastic properties and buckling of silicon nanowires. Adv. Mater. 2008, 20,
3919-3923.

[31] Gurtin, M. E.; Murdoch, A. I. A continuum theory of elastic material surfaces. Arch. Ration. Mech. Anal. 1975, 57, 291-323.

[32] Cammarata, R. C. Surface and interface stress effects in thin films. Prog. Surf. Sci. 1994, 46, 1-38.

[33] Gibbs, J. W. In The Scientific Papers of J. Willard Gibbs; Longmans-Green: London, 1906; Vol. 1, pp 55.

[34] Lu, C. S.; Danzer, R.; Fischer, F. D. Fracture statistics of brittle materials: Weibull or normal distribution. Phys. Rev. E 2002, 65, 067102.

[35] Wang, J.; Kulkarni, A. J.; Sarasamak, K.; Limpijumnong, S.; Ke, F. J.; Zhou, M. Molecular dynamics and density functional studies of a body-centered-tetragonal polymorph of $\mathrm{ZnO}$. Phys. Rev. B 2007, 76, 172103. 